\title{
Distinguishing Models for the Pseudo-gap in Cuprate Superconductors by Probing the Spatial Distribution of Impurity States
}

\author{
C. Kübert \\ Instituto de Física Gleb Wataghin, Universidade Estadual de Campinas, 13083-970, Campinas, SP, Brazil
}

Received on 23 May, 2003.

\begin{abstract}
We argue that the spatial distribution of resonant impurity states in underdoped high- $\mathrm{T}_{c}$ superconductors serves as a probe for distinguishing different theoretical models for the pseudogap state. Superconducting pairing fluctuations are characterized by off-diagonal short-range order which distinguishes them from other possible instabilities that could give rise to the pseudogap phenomena. Due to the mixture of particle and hole states in a superconductor an impurity resonant state is composed of both a particle and a hole-like component. On the contrary a state with a gap induced by a particle-hole instability, like a d-density wave (DDW) or spin-density wave (SDW), exhibits no off-diagonal short-range order and consequently a resonant impurity state consists of only one either particle or hole-like component. Furthermore, a charge-spin separated state shows no resonance state at all inside the gap region.
\end{abstract}

One of the most intriguing property of the underdoped high-temperature superconductors (HTSC) is the suppression of single-particle weight around the Fermi energy $[1,2,3]$ for temperatures well above $T_{c}$. As a consequence the electronic behavior of these materials deviates substantially from that of conventional superconductors. The origin of this so-called pseudo-gap state is one of the key problems to be solved in order to understand the underlying microscopic mechanism of the phenomena of HTSC. As various different theoretical proposals for the pseudo-gap have been put forward the need of experimental probes that are able to distinguish between them is of great interest. Here we argue that scanning tunneling microscopy (STM) experiments in underdoped HTSC, that serve as a local probe of impurity states $[4,5]$, can be used in order to differentiate between at least two classes of proposals.

In one class of models the pseudo-gap is ascribed to a precursor pairing amplitude whose phase coherence is destroyed above $T_{c}[6,7]$. Since such a state exhibits off-diagonal short-range order, particle and hole states get mixed and consequently an induced impurity state is composed of both a particle and a hole-like component. On the other hand in models where the origin of the pseudo-gap is due to a different type of instability (DDW, SDW, stripe order etc.) the property of particle and hole mixing is absent and thus an impurity state has only one either particle or hole-like component. This difference in the local electronic density of states (LDOS) around an impurity can be measured by scanning tunneling microscopy. This prediction can be readily used by STM experiments to detecd for the DDW state in the underdoped cuprates.

The local differential conductance measured at bias voltage $V$ by STM is proportional to the local electron density of states. Here we study the LDOS around a magnetic or non-magnetic $(U=0)$ impurity in a system with competing $d$-wave superconducting (DSC) and DDW order. The model Hamiltonian is given by $(\mathbf{Q}=(\pi, \pi))$

$$
\begin{aligned}
\mathcal{H} & =\sum_{\mathbf{k} \sigma} \epsilon_{\mathbf{k}} c_{\mathbf{k} \sigma}^{+} c_{\mathbf{k} \sigma}+\sum_{\mathbf{k}} \Delta_{\mathbf{k}}\left(c_{\mathbf{k} \uparrow}^{+} c_{-\mathbf{k} \downarrow}^{+}+h . c .\right) \\
& +\sum_{\mathbf{k}} i W_{\mathbf{k}}\left(c_{\mathbf{k} \sigma}^{+} c_{\mathbf{k}+\mathbf{Q} \sigma}+\text { h.c. }\right)+\epsilon_{d} \sum_{\sigma} d_{\sigma}^{+} d_{\sigma} \\
& +V \sum_{\mathbf{k} \sigma}\left(c_{\mathbf{k} \sigma}^{+} d_{\sigma}+\text { h.c. }\right)+U d_{\uparrow}^{+} d_{\uparrow} d_{\downarrow}^{+} d_{\downarrow},
\end{aligned}
$$

where $\left.\epsilon_{\mathbf{k}}=2 t\left(\cos k_{x}\right)+\cos k_{y}\right)-4 t^{\prime}\left(\cos k_{x} \cos k_{y}\right)-\mu$ is the dispersion of the conducting electron band with tight binding parameters $t=400 \mathrm{meV}, t^{\prime}=-50 \mathrm{meV}$ and chemical potential $\mu . \Delta_{\mathbf{k}}=\frac{\Delta_{0}}{2}\left(\cos k_{x}-\cos k_{y}\right)$ and $W_{\mathbf{k}}=$ $\frac{W_{0}}{2}\left(\cos k_{x}-\cos k_{y}\right)$ are, respectively, the DSC and DDW order parameters with $\Delta_{0}$ and $W_{0}$ being the corresponding gap amplitudes.

Within a folded Nambu spinor basis

$$
\begin{aligned}
\hat{\psi}_{\mathbf{k}} & =\left(c_{\mathbf{k} \uparrow}^{+}, c_{\mathbf{k}+\mathbf{Q} \uparrow}^{+}, c_{-\mathbf{k} \downarrow}, c_{-\mathbf{k}-\mathbf{Q} \downarrow}\right), \\
\hat{\varphi} & =\left(d_{\uparrow}^{+}, d_{\uparrow}^{+}, d_{\downarrow}, d_{\downarrow}\right)
\end{aligned}
$$

the electronic Green's function of the clean system is represented by the following matrix 


$$
\hat{G}_{0}^{-1}(\mathbf{k}, \omega)=\left(\begin{array}{cccc}
\omega-\left(\epsilon_{\mathbf{k}}-\mu\right) & -i W_{\mathbf{k}} & -\Delta_{\mathbf{k}} & 0 \\
i W_{\mathbf{k}} & \omega-\left(\epsilon_{\mathbf{k}+\mathbf{Q}}-\mu\right) & 0 & \Delta_{\mathbf{k}} \\
-\Delta_{\mathbf{k}} & 0 & \omega+\left(\epsilon_{\mathbf{k}}-\mu\right) & -i W_{\mathbf{k}} \\
0 & \Delta_{\mathbf{k}} & -i W_{\mathbf{k}} & \omega+\left(\epsilon_{\mathbf{k}+\mathbf{Q}}-\mu\right)
\end{array}\right)
$$

while the impurity Green 's function is given by

$$
\begin{array}{r}
\hat{G}_{d}^{-1}(\omega)=\left[\omega \hat{\tau}_{0}-\Sigma_{\sigma}^{d}(\omega) \hat{\tau}_{0}-\left(\epsilon_{d}+\frac{U}{2}\right) \hat{\tau}_{3}\right. \\
\left.+\omega \sum_{\mathbf{k}} \frac{V^{2}}{\omega^{2}-\epsilon_{\mathbf{k}}^{2}-\Delta_{\mathbf{k}}^{2}} \hat{\tau}_{0}\right],
\end{array}
$$

where $\hat{\tau}_{0}$ is the unit matrix and $\hat{\tau}_{\alpha}(\alpha=1,2,3)$ are the Pauli matrices in folded Nambu space. Scattering of quasiparticles from the impurity is described by a T-matrix, $\hat{T}(\omega)$, which is independent of wave vector in the case of s-wave scattering

$\hat{G}\left(\mathbf{r}, \mathbf{r}^{\prime} ; \omega\right)=\hat{G}^{0}\left(\mathbf{r}-\mathbf{r}^{\prime} ; \omega\right)+\hat{G}^{0}(\mathbf{r} ; \omega) \hat{T}(\omega) \hat{G}^{0}(-\mathbf{r} ; \omega)$.

The T-matrix is given by

$$
\hat{T}(\omega)=V \hat{\tau}_{3} \hat{G}_{d}(\omega) V \hat{\tau}_{3} .
$$

In eq.(5) $\Sigma_{\sigma}^{d}(\omega)$ denotes the self-energy of the d-electron due to the Coulomb interaction $U$ and the last term describes the self-energy due to the hybridization of the d-level with the superconducting electrons. In order to calculate the impurity self-energy for finite $U$ we use the modified perturbation theory introduced by Kajueter and Kotliar [8]. Note that for a non-magnetic impurity $U=0$, i.e. the problem is reduced to a exactly solvable Fano-type one.

The LDOS of the conduction electrons around the impurity is given by

$$
N(\mathbf{r}, \omega)=-\frac{1}{\pi} \operatorname{Im} \hat{G}_{11}(\mathbf{r}, \mathbf{r} ; \omega) .
$$

which is given by

$$
\hat{G}(\mathbf{r}, \mathbf{r} ; \omega)=\hat{\mathbf{G}}^{\mathbf{0}}(\mathbf{0}, \omega)+\hat{\mathbf{G}}^{\mathbf{0}}(\mathbf{r}, \omega) \hat{\mathbf{T}}(\omega) \hat{\mathbf{G}}^{\mathbf{0}}(-\mathbf{r}, \omega) .
$$

The density of states is therefore given by

$$
N(\mathbf{r}, \omega)=\mathbf{N}_{\mathbf{d}-\text { wave }}+\mathbf{N}_{\text {impurity }},
$$

$N_{d-\text { wave }}=-\frac{1}{\pi} \operatorname{Im}\left(\sum_{\mathbf{k}} \hat{\mathrm{G}}_{11}^{0}(\mathbf{k}, \omega)\right)$ denotes the density of states for a clean d-wave superconductor and $N_{\text {impurity }}=$ $-\frac{1}{\pi} \operatorname{Im}\left(\sum_{\mathbf{k}} \mathrm{e}^{\mathrm{i} \mathbf{k} \cdot \mathbf{r}} \hat{\mathrm{G}}^{0}(\mathbf{k}, \omega) \hat{\mathbf{T}}(\omega) \sum_{\mathbf{k}} \mathbf{e}^{-\mathbf{i k} \cdot \mathbf{r}} \hat{\mathbf{G}}^{\mathbf{0}}(\mathbf{k}, \omega)\right)_{11}$

the contribution to the density of states of the conduction electrons due to the impurity. The general form for the density of states due to the impurity is given by

$$
\begin{aligned}
N_{\text {impurity }}=-\frac{1}{\pi} \operatorname{Im} & \left(G_{11}^{0}(\mathbf{r}, \omega) \mathbf{T}_{\mathbf{1 1}}(\omega) \mathbf{G}_{\mathbf{1 1}}^{\mathbf{0}}(-\mathbf{r}, \omega)\right. \\
& +G_{12}^{0}(\mathbf{r}, \omega) \mathbf{T}_{\mathbf{2 1}}(\omega) \mathbf{G}_{\mathbf{1 1}}^{\mathbf{0}}(-\mathbf{r}, \omega) \\
& +G_{11}^{0}(\mathbf{r}, \omega) \mathbf{T}_{\mathbf{1 2}}(\omega) \mathbf{G}_{\mathbf{2 1}}^{\mathbf{0}}(-\mathbf{r}, \omega) \\
+ & \left.G_{12}^{0}(\mathbf{r}, \omega) \mathbf{T}_{\mathbf{2 2}}(\omega) \mathbf{G}_{\mathbf{2 1}}^{\mathbf{0}}(-\mathbf{r}, \omega)\right)
\end{aligned}
$$

In the case of a d-wave superconductor the T-matrix is diagonal; i.e. $T_{12}=T_{21}=0$. For an s-wave state however the diagonal parts do not vanish and are important in order to fulfill the Anderson theorem in the case of a non-magnetic impurity.

The impurity induced resonant states in the gap are given by the poles of the T-matrix. First we focus on the case of a non-magnetic impurity like $\mathrm{Zn}$. In this case the correlation $U=0$ and the Anderson impurity model reduces to that of a potential scatterer. The poles of the T-matrix are given by the zeros of determinant of the inverse impurity Green's function

$$
\left(\omega-\epsilon_{d}-V^{2} \alpha(\omega)\right)\left(\omega+\epsilon_{d}-V^{2} \beta(\omega)\right)-V^{4} \gamma^{2}(\omega)=0,
$$

where

$$
\begin{aligned}
\alpha(\omega) & =\sum_{\mathbf{k}} \frac{\omega-\epsilon_{\mathbf{k}}}{\omega^{2}-E_{\mathbf{k}}^{2}} \\
\beta(\omega) & =\sum_{\mathbf{k}} \frac{\omega+\epsilon_{\mathbf{k}}}{\omega^{2}-E_{\mathbf{k}}^{2}} \\
\gamma(\omega) & =\sum_{\mathbf{k}} \frac{\Delta_{\mathbf{k}}}{\omega^{2}-E_{\mathbf{k}}^{2}}
\end{aligned}
$$

For a d-wave superconductor $\gamma(\omega)=0$ and thus depending on the parameters $V$ and $\epsilon_{d}$ one gets a solution for the above equation. In the case of an s-wave superconductor however $\gamma(\omega) \neq 0$ and thus no solution to the above equation exists. For the s-wave case there exists therefore 
no impurity induced resonant state in the gap, which is in agreement with the Anderson theorem for non-magnetic impurities in a s-wave superconductor.

In Fig. 1 we show the local density of states for a nonmagnetic impurity as Zink in a d-wave superconductor. At the impurity site a resonant state with energy $\omega=-0.05 t$ is obtained. Due to particle-hole mixing in a superconductor a corresponding hole-like component at opposite energy $\omega=0.05 t$ is obtained at the nearest neighboring site along the crystal b-axis.

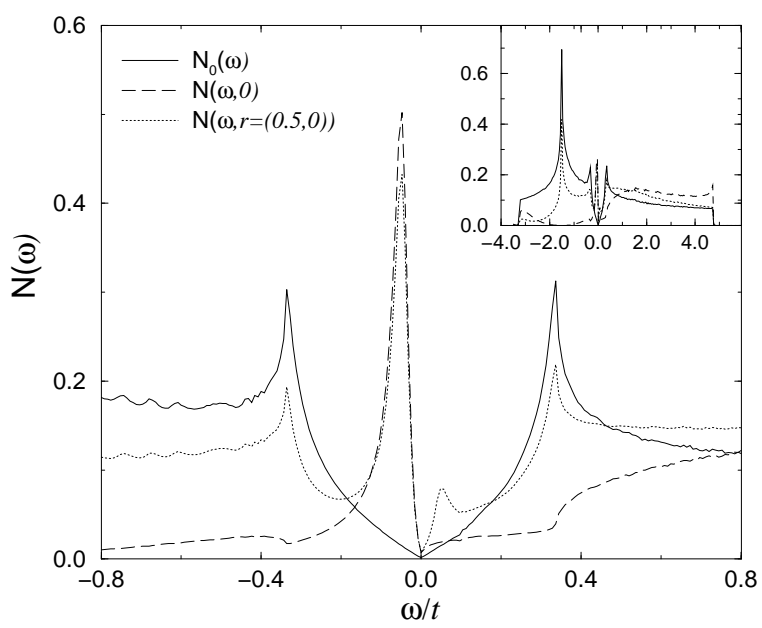

Figure 1. Density of states for a non-magnetic impurity in a d-wave superconductor: full line: d-wave gap without impurity, dashed line: at the impurity site, dotted line: at the nearest neighboring site along the $\mathrm{x}$-axis. Inset: same but for full bandwidth.

For a magnetic impurity $U=4.0 t$ we obtain two resonant impurity states as shown in Fig. 2 for two different hybridization matrix elements $V$.

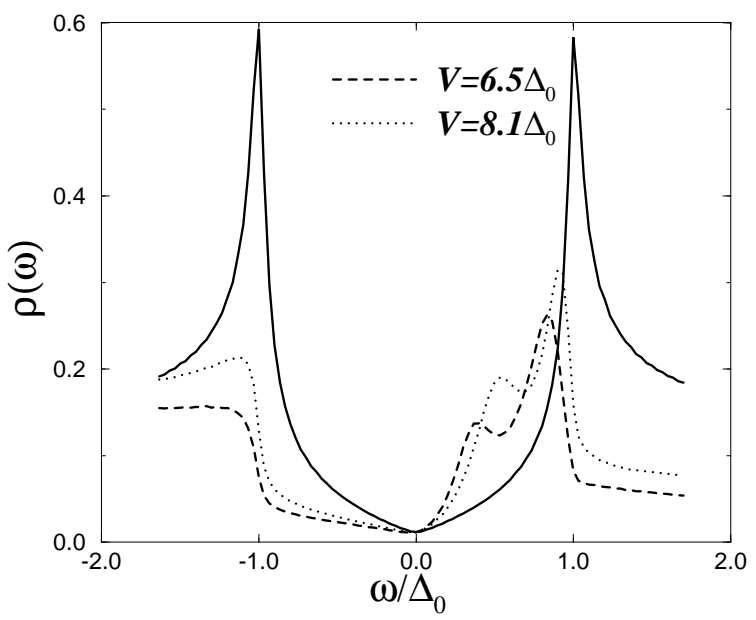

Figure 2. Density of states for a magnetic impurity in a d-wave superconductor with $U=4 t$ and two different values of hybridization matrix element $V$ : full line: without impurity, dashed line: $V=6.5 \Delta_{0}$, dotted line: $V=8.1 \Delta_{0}$.
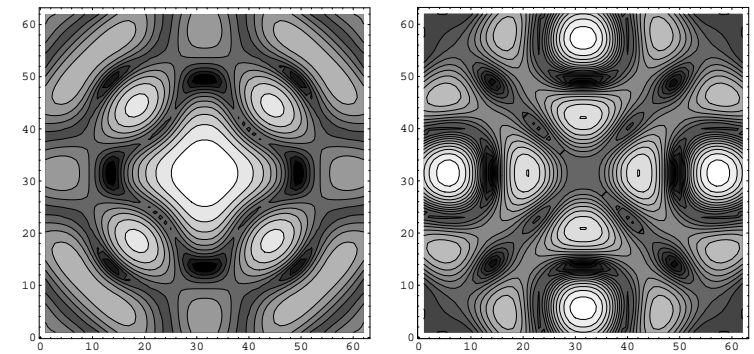

Figure 3. Contour plot of the resonant impurity state in a d-wave superconductor: left: particle component $\omega=0.8 \Delta_{0}$, right: hole component $\omega=-0.8 \Delta_{0}$.

Focusing on the resonant state at higher energy, we show in Fig.3. a contour plot of the particle $\omega=0.8 \Delta_{0}$ and the hole component $\omega=-0.8 \Delta_{0}$. A good agreement with experimental results of Hudson et al. [5] on the density of states around a Ni impurity in BSCCO is obtained.

Next we discuss the result for a DDW state. Due to the absence of particle-hole mixing in this case a resonant impurity state consists of only a particle component at energy $\omega=-0.05 t$, as can be seen in Fig.4. The absence of the corresponding hole-like component is a drastic difference to the case of a d-wave superconductor. Therefore mapping of the resonant state is a power-full tool in order to distinguish these different states. A corresponding contour plot of the impurity state at the resonance energy $\omega=-0.05 t$ shows a completely different spatial structure as demonstrated in Fig.5. Furthermore at the opposite energy $\omega=0.05 t$ no structure at all is observed, since no hole-like component exists.

We now discuss the interesting case of coexisting DDW and d-wave superconducting state. In the following we assume that the superconducting critical temperature $T_{c}$ is lower than the corresponding critical temperature for the DDW state $T_{D D W}$.

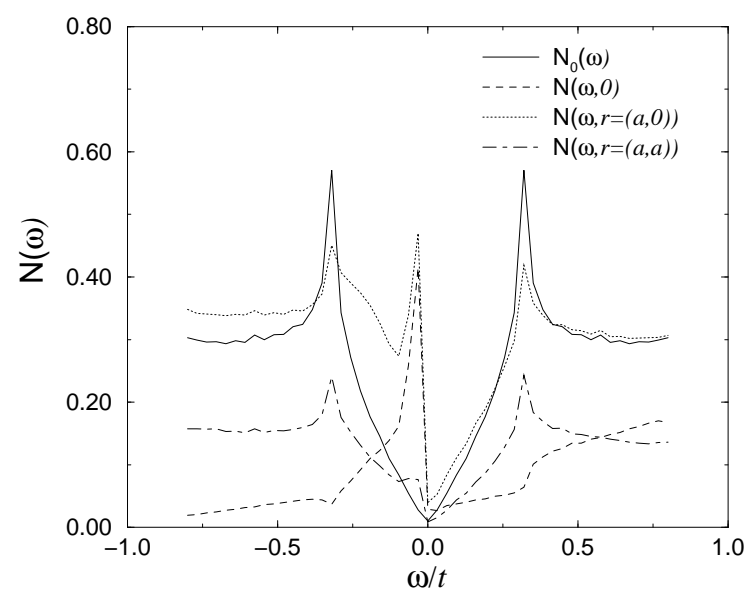

Figure 4. Density of states for a non-magnetic impurity in a DDW state: full line: d-wave gap without impurity, dashed line: at the impurity site, dotted line: at the nearest neighboring site along the $\mathrm{x}$-axis, dotted-dashed line: at the nearest neighboring site along the diagonal of the crystal lattice. 


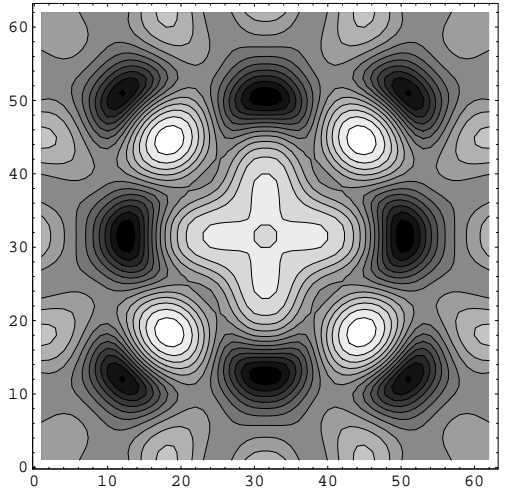

Figure 5. Contour plot of the resonant impurity state in a DDW state. Only a particle component exists.

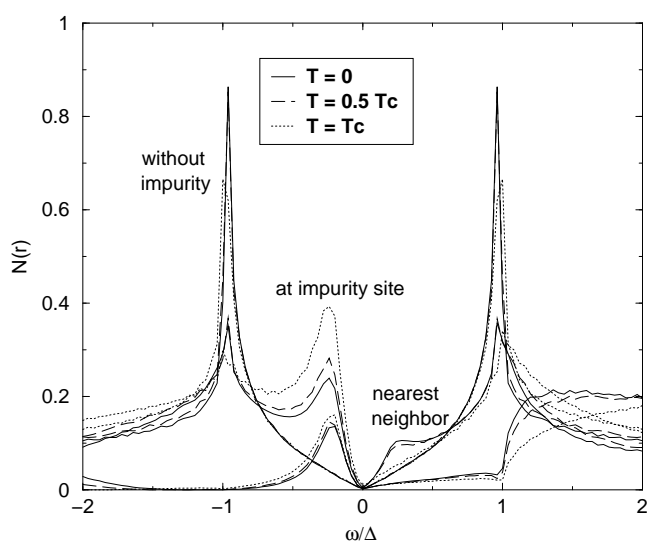

Figure 6. Temperature effect on the resonant impurity state: in a d-wave superconductor coexisting with a DDW state: full line: without impurity, dashed line

Below the critical temperature $T_{c}$ we again get a particle and a hole-like component of the resonant state as shown in Fig.6. If we now raise the temperature above $T_{c}$ but still below $T_{D D W}$, e.g. $T_{c}<T<T_{D D W}$, we observe that the hole-like component at he nearest neighboring site vanishes. At the same time the particle-like component increases, due to the transfer of spectral weight from the disappearing hole component. This effect should be clearly visible in a STM measurement.

As we have shown above different states will have different signatures of the resonant impurity state and thus can in principle be distinguished by a STM experiment. Unfortunately, until now experiments on resonant impurity states have only been conducted on optimally doped samples of BSCCO $[4,5]$, where no pseudo gap is present. Since understanding the pseudo-gap is considered the key for the theory of HTSC, a corresponding STM experiment on underdoped samples with a very low $T_{c}$ and a huge pseudo-gap is in demand. In fact such an experiment would help to distinguish the different theoretical models proposed for the pseudo-gap in the literature.

However, special care has to be taken of what type of impurity one should study. As was shown by Pan et al.[4] a nonmagnetic impurity as $\mathrm{Zn}$ locally destroys the superconductivity, as indicated by the absence of a hole-like resonance. Nevertheless a particle-like resonance is observed, due to the depletion of states near the Fermi level, which we attribute to the pseudo-gap. On the other hand a Ni impurity preserves the superconducting state and thus also a hole-like component is observed due to superconducting fluctuations [5].

Therefore we propose a STM experiment in the underdoped region of HTSC with embedded Ni impurities at temperatures above $T_{c}$. The absence of a hole-like component of a resonant state together with the existence of a particlelike component would speak against the precursor pairing mechanism and be in favor of a particle-hole state such as a DDW state. The complete absence of any resonance in the pseudo-gap phase however, would demonstrate that a more exotic type of state like for example a spin charge separated state is realized. As a last remark we would like to comment that a promising achievement on the experimental side towards this direction was recently obtained by Y. Kohsaka et al.[9] who were able to synthesized $\mathrm{Na}$-doped $\mathrm{Ca} 2 \mathrm{CuO} 2 \mathrm{Cl} 2$ single crystals with well defined surfaces in the extreme underdoped regime.

\section{References}

[1] A.G. Loeser et al., Science 273, 325 (1996).

[2] H. Ding et al., Nature 382, 51 (1996).

[3] Ch. Renner et al., Phys. Rev. Lett. 80, 149 (1998).

[4] E.W. Hudson et al., Science 285, 88 (1999); S.H. Pan et al., Nature, 403, 746 (2000).

[5] E.W. Hudson et al., Nature, 411, 920 (2001).

[6] V.J. Emery and S.A. Kivelson, Nature (London) 374, 434 (1995).

[7] M. Franz and A.J. Millis, Phys. Rev. B 58, 14572 (1998); H.-J. Kwon and A.T. Dorsey, ibid 59, 6438 (1999).

[8] H. Kajueter and G. Kotliar, Phys. Rev. Lett. 77, 131 (1996).

[9] Y. Kohsaka et al., cond-mat/0209339; F. Ronning et al., condmat/0301024. 